\title{
Electrocoagulación de aguas residuales de la industria láctea
}

\section{(Dairy industry wastewater electrocoagulation)}

\author{
Pamela López ${ }^{1}$, Antonio Harnisth ${ }^{1}$
}

\begin{abstract}
Resumen:
La industria láctea genera gran cantidad de agua residual en sus procesos, estos desechos líquidos son vertidos directamente a fuentes hídricas, sin ningún tratamiento previo. Para dar una solución a este problema, se estudió una técnica que permite el tratamiento de este tipo de aguas con alta carga orgánica, se la conoce como electrocoagulación. Para llevar a cabo el proceso de electrocoagulación se determinó tres factores que son: tiempo, número de placas del electrocoagulador y voltaje del sistema. Una vez realizadas las corridas experimentales se evidencio la eficiencia del tratamiento de electrocoagulación, obteniendo como porcentajes de remoción de aproximado de DQO fue de $93 \%$, DBO5 de $82 \%$ y de SS de $76 \%$.
\end{abstract}

Palabras clave: electrocoagulación; turbidez; eficiencia; DQO; DBO.

\begin{abstract}
:
The dairy industry generates lots of water discharge processes, these wastewater are discharged directly into water sources without any pretreatment. . In order to solve this problem there is a treating technique called electrocoagulation that treat of waste water with high organic load. There are three factors to carry out the electrocoagulation process as: time, number of plates and system voltage. After completing the set up and running the experiment, the electrocoagulation treatment efficiency was evident, as the approximate percentage of COD removal was $93 \%, 82 \%$ of $\mathrm{BOD}_{5}$ and $76 \%$ of SS.
\end{abstract}

Keywords: electrocoagulation; turbidity; efficiency; COD; BOD.

\footnotetext{
${ }^{1}$ Universidad de las Américas, Quito - Ecuador (\{gplopezl, o.harnisth\} @udlanet.ec)
} 


\section{Introducción}

La industria láctea representa una de las principales fuentes de contaminación de los recursos hídricos, ya que en sus procesos de producción se utiliza aproximadamente entre 1,3-3,2 L de agua/kg de leche recibida (Gandarillas, Sánchez y Serrano, 2009). Entre las operaciones que generalmente producen vertidos están: el lavado y esterilización de los depósitos, tanques, equipos de enfriamiento y suelos. Prácticamente el agua que se emplea en la elaboración de estos productos es para la limpieza de los utensilios y el área de trabajo (Nemerow y Dasgupta , 2009).

Del 90 al $95 \%$ aproximadamente del DBO presente en las aguas residuales de la industria láctea, se debe a la pérdida de leche o productos lácteos en los procesos de elaboración. Los productos químicos limpiadores empleados en los procesos de lavado, se traducen en la presencia de DQO (Arango et al., 2008).

El consumo descontrolado y los altos niveles de contaminación del recurso agua, han dado lugar a la investigación de diversas tecnologías con el fin de reducir la carga orgánica e inorgánica que se encuentran presentes en las aguas residuales, una de ella es la electrocoagulación, proceso en el cual son desestabilizadas las partículas de contaminantes que se encuentran suspendidas, emulsionadas o disueltas en un medio acuoso, induciendo corriente eléctrica en el agua a través de placas metálicas paralelas de diversos materiales, siendo el hierro y el aluminio los más utilizados (Chen, 2004).

El objetivo de esta investigación fue el diseño de un proceso de electrocoagulación para el tratamiento de aguas residuales de la industria del yogurt. Mediante la determinación de los parámetros de operación en cuanto al tiempo, voltaje y número de placas. Para finalmente evaluar la eficiencia del proceso en la remoción de la demanda química de oxígeno (DQO), demanda bioquímica de oxígeno (DBO) y sólidos suspendidos (SS); y así determinar el sistema óptimo de operación.

\section{Metodología}

\subsection{Muestreo y caracterización del agua residual}

Las muestras tomadas para el tratamiento de electrocoagulación fueron realizadas antes de ser descargadas al alcantarillado. Se tomó 3 canecas de plástico de 20 litros aproximadamente, cada vez que era necesario para los tratamientos de la investigación. 
Debido a que el flujo de agua residual no es continuo, se decidió tomar muestras simples. Una vez tomadas las muestras del punto de muestreo escogido, fueron llevadas a refrigeración inmediatamente para que se preserven hasta el momento de su uso.

La muestra de agua residual tomadas para el análisis de los parámetros físico-químicos en un laboratorio certificado, se la realizó en una botella de vidrio de $500 \mathrm{ml}$. En tanto que la muestra que se analizó en el Laboratorio de Ingeniería Ambiental-UDLA, se tomó en una botella plástica de 3 litros.

La caracterización del agua cruda se realizó en base a los parámetros establecidos en la Guía de Parámetros Mínimos por Sector Productivo dado por la Clasificación Internacional Industrial Uniforme (CIIU), establecidos en la Ordenanza 404, Resolución N0002.

Los parámetros como $\mathrm{pH}$ y temperatura se realizaron in situ en el momento del muestreo. Los análisis de $\mathrm{DBO}_{5}$, SSed y SS se los realizó en el Laboratorio de Ingeniería Ambiental. Para analizar los aceites y grasas y el DQO se prescindió de los servicios de un laboratorio certificado por la OAE.

\subsection{Diseño Experimental}

Se utilizó para esta investigación el diseño factorial $2^{3}$, mediante la utilización del software Minitab 17. Se analizó los factores: tiempo, número de placas del electrocoagulador y voltaje del sistema, con el fin de conocer el tiempo óptimo de tratamiento, además de estudiar los efectos entre el número de placas utilizadas y la fuente de energía disponible.

Tabla 1. Factores de diseño, con sus dos niveles de estudio

\begin{tabular}{|c|c|c|}
\hline Factores & Nivel (-1) & Nivel (1) \\
\hline Tiempo & $30 \mathrm{~min}$ & $60 \mathrm{~min}$ \\
\hline Número de Placas & 6 & 12 \\
\hline Voltaje & 6 voltios & 12 voltios \\
\hline
\end{tabular}

El orden de los experimentos llevados a cabo se realizó completamente al azar, con la interacción de los factores estudiados con cada uno de sus niveles de estudio. Para el presente estudio se tomó como base tres factores de operación: tiempo de reacción, número de placas y voltaje; con dos niveles cada uno. La variable de respuesta fue la disminución de la turbidez del agua tratada, tomando como referencia la turbidez del agua residual. 
Los diferentes tratamientos del diseño experimental, se realizaron en un electrocoagulador a escala laboratorio, donde se trataron 9 litros de agua residual. Una vez determinados los parámetros óptimos de operación del electrocoagulador a escala laboratorio, se procedió a la construcción del electrocoagulador a escala piloto para el tratamiento de 33 litros de agua, con los factores de mayor significancia del diseño experimental.

\section{Resultados}

En la figura 1, se observa que el tiempo de reacción y el voltaje interactúan afectando la respuesta de turbidez. El mejor resultado para la disminución de turbidez se encuentra en la parte superior derecha del cubo, es decir, que la relación entre el número de placas, el tiempo de reacción y el voltaje en su nivel alto respectivamente, permiten una disminución de 13.45 NTU frente a la turbidez inicial de 270 NTU. Otro dato significativo es la turbidez de 15.65 NTU, en donde interactúan el tiempo de reacción en su nivel alto (1), el voltaje en su nivel bajo (1) y el número de placas en el nivel alto (1).

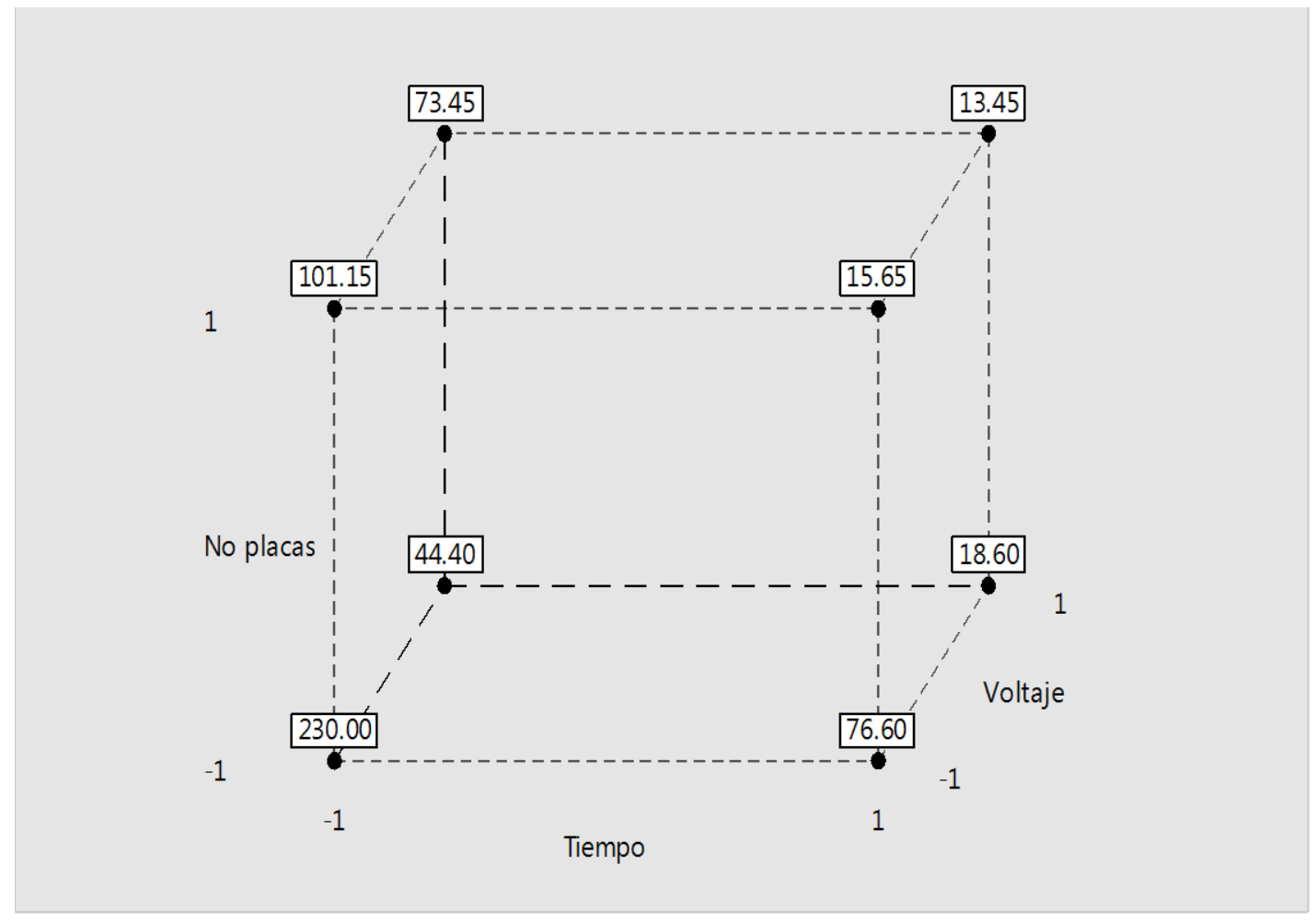

Figura 1. Gráfica de cubos (medias de los datos) para Turbidez.

La Figura 2, indica que tanto el tiempo de reacción y el voltaje tienen efectos similares sobre la turbidez, ya que ambos factores presentan similitud en su pendiente al estar más inclinadas. La disminución en la turbidez para estos dos factores se da cuando pasan de su nivel bajo al nivel alto afectando directamente la variable de respuesta. Se puede concluir que la saturación de cargas positivas que impediría la aglomeración de los coloidales y la precipitación de los sedimentos. La saturación de cargas negativas, al contrario generaría el aumento de sólidos de gran tamaño incapaces de precipitar. 


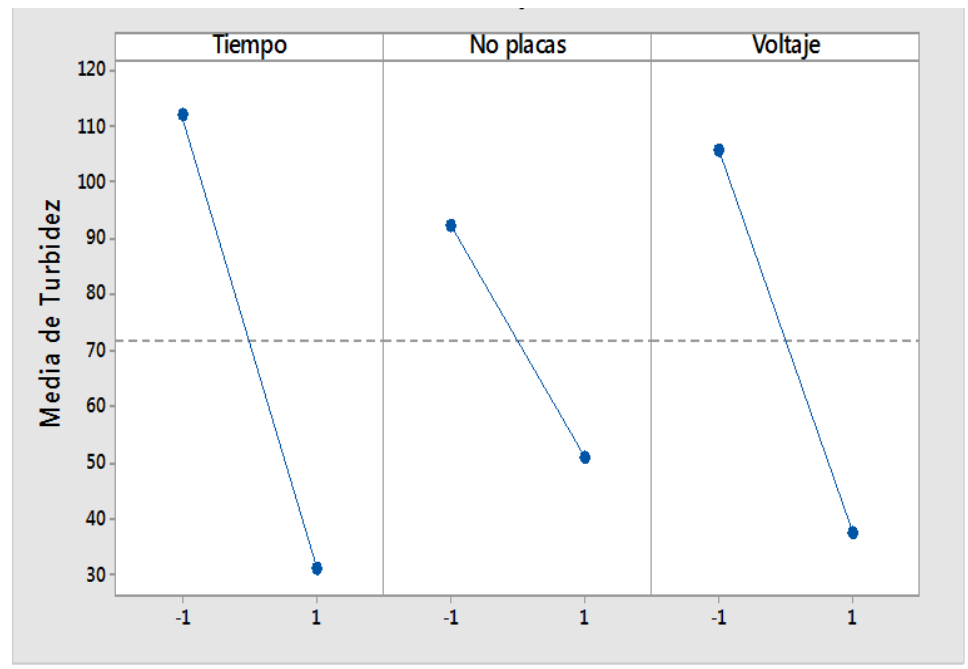

Figura 2. Gráfica de efectos principales para Turbidez (medias ajustadas)

En la figura 3, el mejor resultado en cuanto a la disminución de la turbidez se da cuando el factor tiempo se encuentra en su nivel alto, en tanto que no se presenta mayor incidencia en la variable de respuesta con el factor número de placas, es decir no se representa una interacción significativa al pasar de un nivel a otro en el factor número de placas.

Entre el tiempo de reacción y el voltaje se observa que existe un efecto significativo en la disminución de la turbidez cuando los dos factores interactúan pasando del nivel bajo al alto, presentando mejores resultados en el nivel alto tanto para el tiempo como para el voltaje.

La relación entre los factores número de placas y voltaje, se observa que existe mayor incidencia en la diminución de la variable de respuesta cuando el voltaje está en su nivel alto, en tanto que el número de placas no afecta directamente a la variable de respuesta indistintamente del nivel.

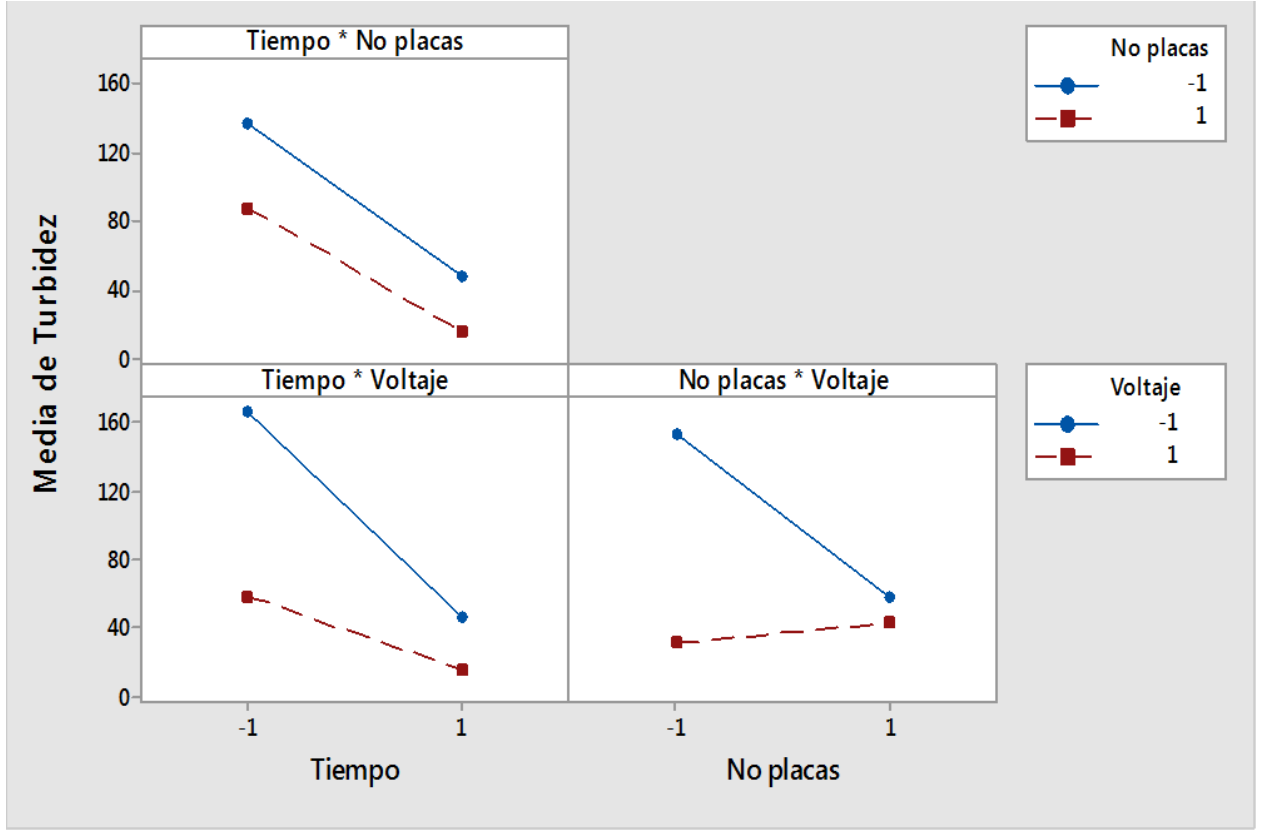

Figura 3. Gráfica de interacción para Turbidez (medias ajustadas) 
En la figura 4, se muestra dos vasos de precipitación, el uno contiene el agua residual producto de la elaboración de yogurt y la segunda el agua tratada después de haber sido sometida a la electrocoagulación.

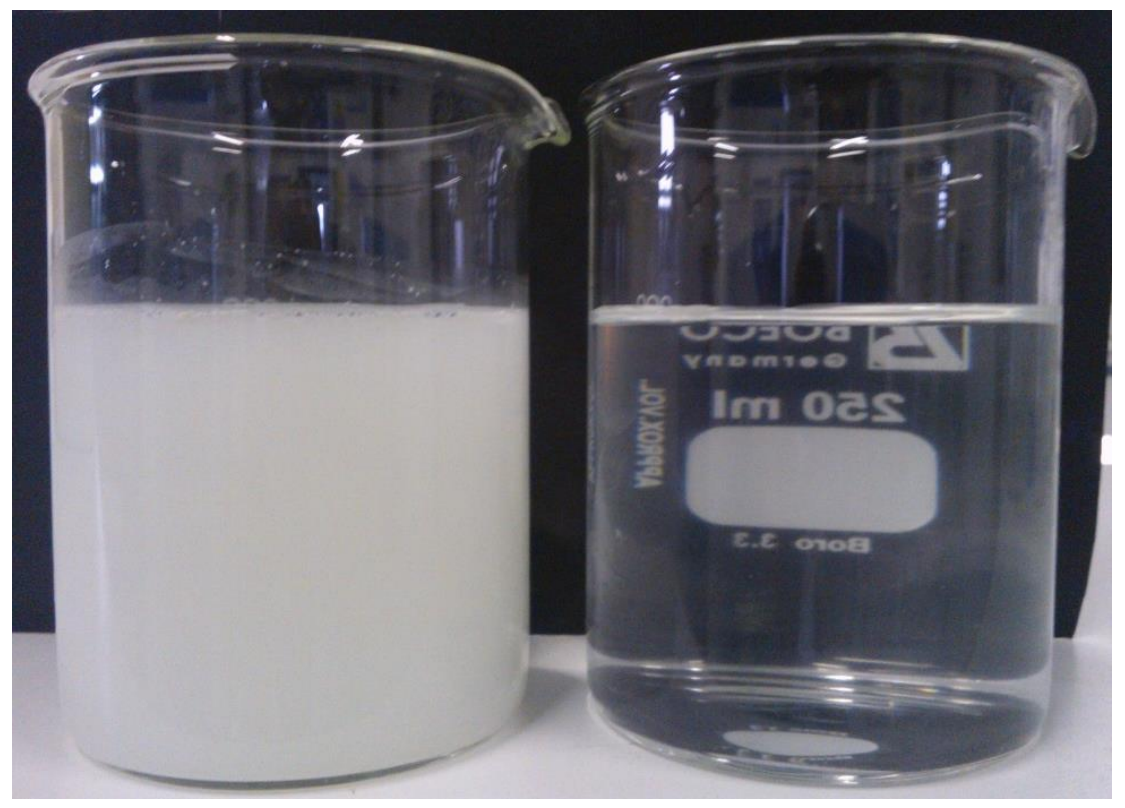

Figura 4. Agua residual antes y después del tratamiento.

\section{Discusión}

Los parámetros escogidos para el análisis de la eficiencia del sistema de tratamiento propuesto fueron: DBO, DQO y SS.

En la figura 5, A. se observa los resultados obtenidos en los análisis de $\mathrm{DBO}_{5}$ antes y después de la electrocoagulación. En donde se aprecia que existe aproximadamente un promedio de eficiencia del $93.33 \%$ de remoción de $\mathrm{DBO}_{5}$, entre los $420 \mathrm{mg} / \mathrm{l}$ de $\mathrm{DBO}_{5}$ inicial y de $28 \mathrm{mg} / \mathrm{l}$ después del tratamiento de las tres réplicas.

B. el resultado inicial y los datos finales existe una reducción de $860.5 \mathrm{mg} / \mathrm{l}$ de DQO aproximadamente, que representa alrededor de un $82.42 \%$ de remoción de este parámetro, promedio entre el agua tratada M1 y M2. El proceso de electrocoagulación permite que tanto el $\mathrm{DBO}_{5}$ como el $\mathrm{DQO}$ se reduzcan considerablemente, ya que las cargas positivas producto de la oxidación del $\mathrm{Al}$, se liberan y se dispersan en el medio líquido; estos iones metálicos tienden a formar óxidos metálicos que atraen eléctricamente a los contaminantes que han sido desestabilizados (Callejas y Prieto, 2011, p. 203).

C. el agua cruda tiene un $24.3 \mathrm{mg} / \mathrm{l}$ de sólidos suspendidos, después del tratamiento los resultados de las muestras $\mathrm{M} 1, \mathrm{M} 2$, y $\mathrm{M} 3$ indican una reducción de SS de aproximadamente un $76.81 \%$. Esto se debe a que los sólidos en suspensión que se producen logran alcanzar la densidad adecuada para su floculación. La eliminación de las materias por sedimentación se basa 
en la diferencia de peso específico entre las partículas sólidas y el líquido donde se encuentran, que acaba en el depósito de las materias en suspensión (Ramalho., 2003, p 92).

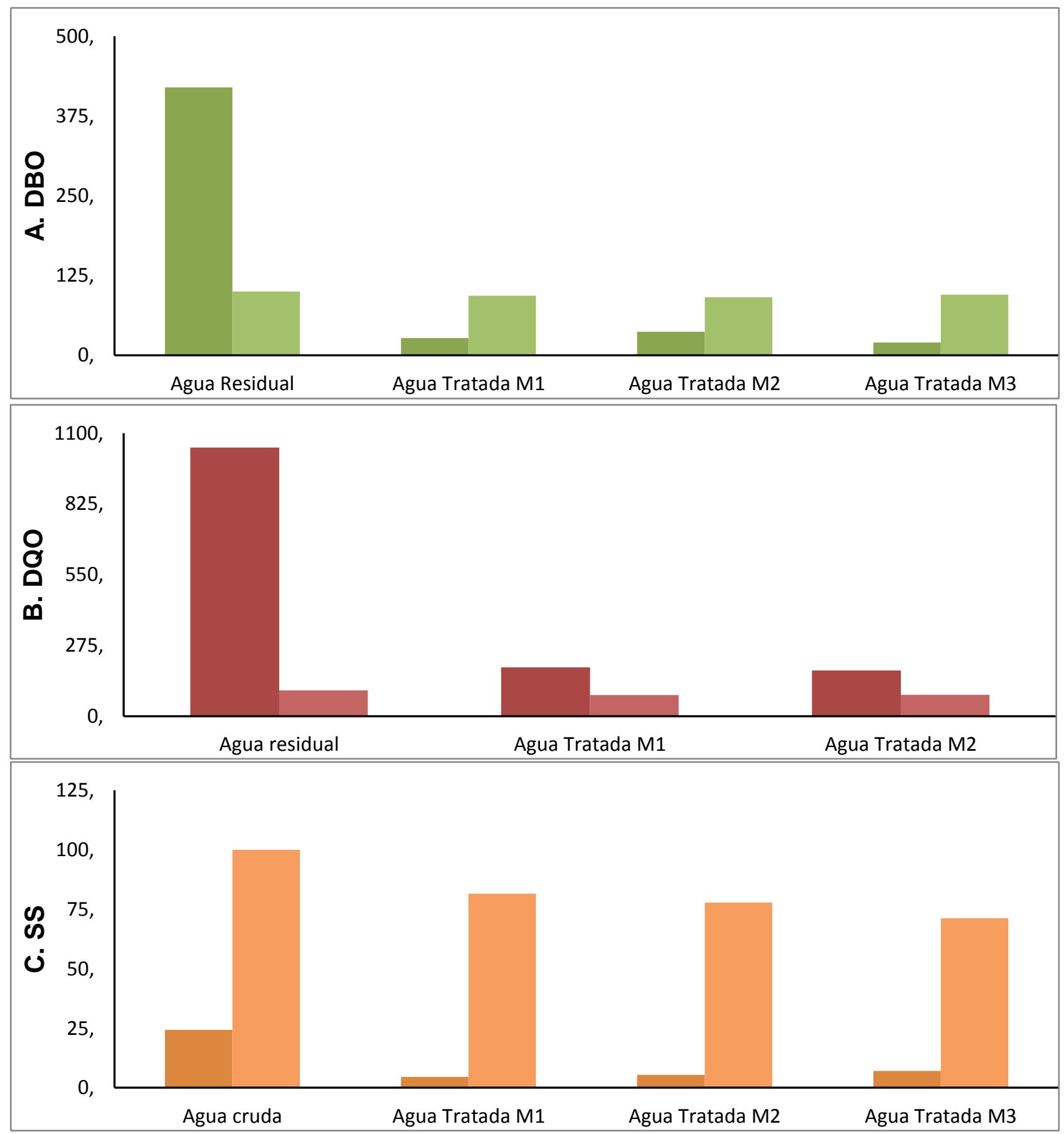

Figura 5. Gráficas de porcentajes de remoción.

A. DBO: Agua residual, Agua tratada M1, Agua tratada M2 y Agua tratada M3

B. DQO: Agua residual, Agua tratada M1, Agua tratada M2

C. Sólidos suspendidos: Agua cruda, Agua tratada M1, Agua tratada M2 y Agua tratada M3 
De la investigación se puede concluir, la saturación de cargas positivas que impediría la aglomeración de los coloidales y la precipitación de los sedimentos. La saturación de cargas negativas, al contrario generaría el aumento de sólidos de gran tamaño incapaces de precipitar. El aumento en el tiempo de electrolisis permite cambios notorios en la reducción de aceites y grasas. Para estudiar el efecto del tiempo en la eficiencia del proceso hay que tomar en cuenta la densidad de corriente y el $\mathrm{pH}$.

El proceso se da gracias al desprendimiento de los iones de aluminio, con carga positiva, que desestabilizan las cargas negativas de los coloidales, permitiendo la aglomeración de los sólidos en suspensión, afectando directamente con la turbidez del agua tratada.

El aumento del tiempo de reacción permite una mayor formación del hidróxido del metal, que promueve la remoción de los sólidos coloidales y de DQO. Por medio de la formación de flóculos que pueden precipitar fácilmente (Karichappan, Venkatachalam y Jeganathan, 2014, p. 7-8).

\section{Conclusiones y Recomendaciones}

Se construyó un electrocoagulador a escala piloto para llevar acabo los diferentes ensayos, con materiales de bajo costo y de fácil acceso en el mercado. De los factores analizados: tiempo, voltaje y número de placas, en el diseño experimental, se concluye que los tres influyen significativamente en el proceso de electrocoagulación. Sin embargo el tiempo de reacción y el voltaje tienen mayor incidencia sobre los mejores resultados de remoción de la variable estudiada. La turbidez inicial de 770 NTU, disminuyó aproximadamente a 19,9 NTU después del tratamiento de estudio.

El factor número de placas influye en el estudio ya que a mayor número de placas existen mejores resultados. A menor distancia entre placas hay una mejor formación de flócs, es decir la distancia es inversamente proporcional a la formación de flóculos residuales. Este se debe a que entra las placas existe una mayor movilidad de los iones desprendidos durante la electrolisis.

En los diferentes ensayos llevados a cabo se pudo determinar que la temperatura y el pH, no varían significativamente después de cada tratamiento, en comparación con los datos iniciales. La formación de burbujas de gas de $\mathrm{H}_{2}$ en la superficie de los electrodos, permite que las partículas desestabilizadas en el proceso, queden suspendidas y se puedan separar con mayor facilidad. Al terminar el tratamiento de electrocoagulación, el agua presenta una remoción significativa en la disminución de $\mathrm{DQO}, \mathrm{DBO}_{5}$ y SS.

A partir de esta investigación a escala piloto, se recomienda la aplicación industrial del proceso de electrocoagulación, para la validación de las variables estudiadas. Además de la importancia de continuar con el análisis de otros factores de influencia en esta propuesta de estudio. El análisis 
de la cantidad de aluminio residual que puede contener el agua tratada, y algún tratamiento posterior a la electrocoagulación, que permita la remoción de Al.

La utilización de otro tipo de metal en los electrodos, tanto para el ánodo como para el cátodo, para conocer si existe mayor eficiencia con otro tipo de material.

Realizar investigaciones sobre este proceso con otro tipo de aguas residuales de diferentes industrias.

\section{Bibliografía}

Arango, A. y Garcés, L. (2008). Tratamiento de aguas residuales de la industria láctea por electrocoagulación. Recuperado el 8 de abril de 2014 de http://www.inese.es/html/files/pdf/amb/iq/458/14ARTICULOABR.pdf

Callejas, J. y Prieto, F. (2011). Depuración por electrocoagulación en un lactosuero: cinética del proceso. Querétaro, México: Universidad Autónoma del Estado de Hidalgo.

Chen, G. (2004). Electrochemical technologies in wastewater treatment. In: Separation and Purification Technology. Recuperado el 10 de enero de 2014 de http://www.jpcomplex.ir/content/media/image/2013/08/772_orig.pdf

Gandarillas L., Sánchez T. y Serrano R. (2009). Estación depuradora de aguas residuales de una industria láctea. Recuperado el 28 de Julio de 2014 de http://api.eoi.es/api_v1_dev.php/fedora/asset/eoi:36163/componente36162.pdf

Karichappan, T., Venkatachalam, S. y Jeganathan, P. (2014). Optimization of electrocoagulation process to treat grey wastewater in batch mode using response surface methodology. Recuperado el 25 de Julio de 2014 de http://www.ijehse.com/content/12/1/29

Nemerow, N. y Dasgupta, A. (2009). Tratamiento de vertidos industriales y peligrosos. Madrid, España: Ediciones Díaz de Santos, S.A.

Ramalho R. S. (2003). Tratamiento de aguas Residuales. Madrid, España. Editorial Reverté S.A.

Sengil, A., Ozacar, M., (2006). Treatment of dairy wastewaters by electrocoagulation using mild steel electrodes. Journal of Hazardous Materials. 